JOURNAL DE PHYSIQUE IV

Colloque C2, supplément au Journal de Physique III, Volume 5, février 1995

$\mathrm{C} 2-53$

\title{
Dynamical Lattice State at the Initial Stage of Martensitic Transformation and Possibilities of its Physical Realization
}

\author{
V.V. Letuchev, S.V. Konovalov and M.P. Kashchenko \\ Professor M.P. Kashchenko, Holder of the Physics Chair, Ural State Wood Technology Academy, \\ Sybirskyi Trakt, 37, 620032 Ekaterinburg, Russia
}

\begin{abstract}
Principles of the physical simulation of conditions at the initial stage of $\gamma-\alpha$ martensitic transformation are formulated on the basis of the theory which synthesizes conceptions of the heterogeneous nucleation and the wave growth of martensite. The choice of the experimental method avallable to realize the artificlal initiation of transformation is Justified. Possible mechanisms of excitation of the hypersonic controliing waves by a single supershort laser pulse are presented. The Information about perspectives of using the recelved results for alloys featuring the shape-memory effect is also presented.
\end{abstract}

\section{TNERODUCTION}

The physical realization of conditions corresponding to the initial stage of martensitic transformation (MT) in the original austenite lattice means (in the context of present communication) the artificial creation of object similar to the martensite nucleus. The following growth of the last one will cause the formation of macroscopic martenSit1c crystal (MC) in the austenitic volume. Thus by the man-made triggering there may be produced a single martensitic lamella and its dimensions (excepting the thickness) will be defined only by overall dimensions of the investigated specimen. Such object w111 allow to recelve unique refined data about martensite properties. Another important consequence: the method of controlled initiation of MT gives a possibility to form a signal riglaly synchronized in time with the beginning of the martensitic lamella growth. The use of such reference slgnal opens perspectives to investigate in detall the separated stages of the MC and MC ensemble dynamics in the real time immediately during the $\mathrm{M}$ process.

The expertments on the controlled initiation of MT demand a detalled information concerning both properties of the austenite lattice state corresponding to the IT initial stage and mechanisms maintaining the subsequent evolution of this state. Such information may be taken from the theoretical model which describes adequately all the set of MP observed features. The next problem to be resolved is the cholce of experimental method allowing to carry out the controlled inftiation.

\section{2. $\gamma-a$ MARTEUSITIC TRANSFORUATION: PARTICULARITIES AND MBCHANISUS}

In this work the problem of intilation of the single MC growth is 
considered for the $\gamma-\alpha \mathrm{MT}$ in the Fe-31,5\%N1 alloy single-crystals. The original high-temperature $\gamma$-phase (austenite) has fcc-lattice and the low-temperature $\alpha$-phase (martensite) has bcc-lattice [1]. The transformation is characterized by the pronounced attributes of the irst-order phase transition and proceeds as cooperative one. It is important to note that the specific (per unit of mass) volume of $\alpha$-phase is more than one of $\gamma$-phase.

A martensite arises in the form of lamellas with a small ratio of thickness to the other linear dimensions. The lamella thickmess is about $1 \mu \mathrm{m}$. A lamella habit plane (an interphase boundary) takes 24 consistent orientations which are described by collection $\left\{310{ }^{15}\right\}_{\gamma}$ (relative to the crystallographic axis of $\gamma$-phase). The martensite Iamella formation at the MT process is accompanied by appearance of the relief on extemal surfaces that allows to register by opt1cs the transformation. Often the MC set demonstrates the well defined order in the relative position. This particularity allows to say about the formation of $\mathrm{MC}$ ensembles.

MT in the $\mathrm{Fe}-31,5 \% \mathrm{~N} 1$ single-crystals exhibits brightly the athermal macrokinetics and may be classified as "explosive" transformation (a major volume proportion of martensite arises during the explosion). This particularity causes the weIl audible sound click at the moment of transition.

It should be noted specially that $\mathrm{MC}$ at the $\gamma-\alpha$ MT grows at the high rate. In accord with data received experimentally in works $[2,3]$ the magnitude of this rate exceeds the longltudinal sound velocity.

$\gamma-\alpha$ MT obviously demonstrates the attributes of the first-order phase transition which allows to suppose that the stages of nucleation and MC growth take place. The martensite nucleation is considered to have a heterogeneous character and dislocations play the important role at this process.

Whole set of $\gamma-\alpha M T$ observed features may be correctly and consistently described within the scope of the approach which is based on synthesis of two conceptions: of the heterogeneous (near dislocation) nucleation and of the $\mathrm{MC}$ growth controlled by quasilongitudinal displacement waves. Experimental data about high (supersonic) rate of MC growth lead to the wave mechanlsm which is physically unalternat1ve. The indicated approach in the most complete view is expounded in the monograph [4]. The work [5] represents a compact (but enough qualitative) interpretation of basic ldeas and includes the new results and conclusions.

The theory points important both to understand the principles of modelling of lattice state on the MT origin stage and to carry out the MT artificial initiation are presented below.

MT starts from the excited state in the form of heavily elongated rectangular parallelepiped with the long opposite faces oscillating in palrs in antiphase. The orientation of parallelepiped is defined

by the triple of the mutually orthogonal vectors $\vec{\xi}_{1}, \vec{\xi}_{2}, \vec{\xi}_{3}$ (F18.1). The transformation in the paralleleplped volume will have started when amplitudes of oscillations in the directions $\vec{\xi}_{1}$ and $\vec{\xi}_{2}$ w11l reach values $\varepsilon_{1}^{\text {th }}$ and $\varepsilon_{2}^{\text {th }}$ (threshold deformations) and bestdes the deformations w111 satisfy the conditions:

$$
\varepsilon_{1}>0, \varepsilon_{2}<0, \varepsilon_{3}<\varepsilon_{1},\left|\varepsilon_{2}\right|
$$

(tension, compression and small deformation in the airections $\vec{\xi}_{1,2,3}$ ). 
The vector $\vec{\xi}_{3}$ lies in the weak-distorted (invariant if $\varepsilon_{3}=0$ ) plane favorable for the interphase contact. The energy liberated during transformation is spent partly for a generation of two hypersonic wave beams which propagate at the velocities $c_{1}, c_{2}$ (the wave normals: $\left.\mathbf{n}_{1}=c_{1} / c_{1}, n_{2}=c_{2} / c_{2}\right)$. It is easy to see that the intersection Iine (as well as the area where the conditions (1) are fulfilled) of two wave fronts moves at the velocity $\mathbf{c}=\mathrm{c}_{1}+\mathrm{c}_{2}$. As a result the martensitic lamella grows at the velocity c. A mechanism of generation of two longitudinal controliling waves is based on the stimulated acoustic

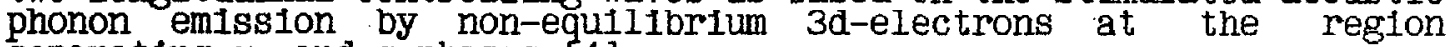
separating $\gamma$ - and $\alpha$-phases [4].

In the case of the spontaneous $\gamma-\alpha$ MT the excited state is considered to arise by pluctuation in the well definite area near the rectilinear dislocation. In so doing, the vectors $\vec{\xi}_{i}$ are the elgenvectors of elastic deformation tensor in the indicated area. The corresponding eigenvalues $\varepsilon_{i}$ satisfies the conditions (1). In this area the energet1c interphase barrier is lowered and the conditions favorable to arise the threshold fluctuation take place.

Synthesis of two conceptions of the heterogeneous nucleation and of the wave growth leads to the additional conditions [5]:

$$
\begin{aligned}
\mathbf{n}_{1} & \approx \vec{\xi}_{1}, \quad n_{2} \approx \vec{\xi}_{2}, \\
c_{2} / c_{1} & \Leftrightarrow\left(\varepsilon_{1} /\left|\varepsilon_{2}\right|\right)^{1 / 2} .
\end{aligned}
$$

It should be noted that $\varepsilon_{1}>\left|\varepsilon_{2}\right|$ (as a result of increase of the specific volume during $\gamma-a$ transformation) and $c_{2}>c_{1}, 1 . e$. the compression deformation in the transformed region is effected by the ware propagating at the lower velocity.

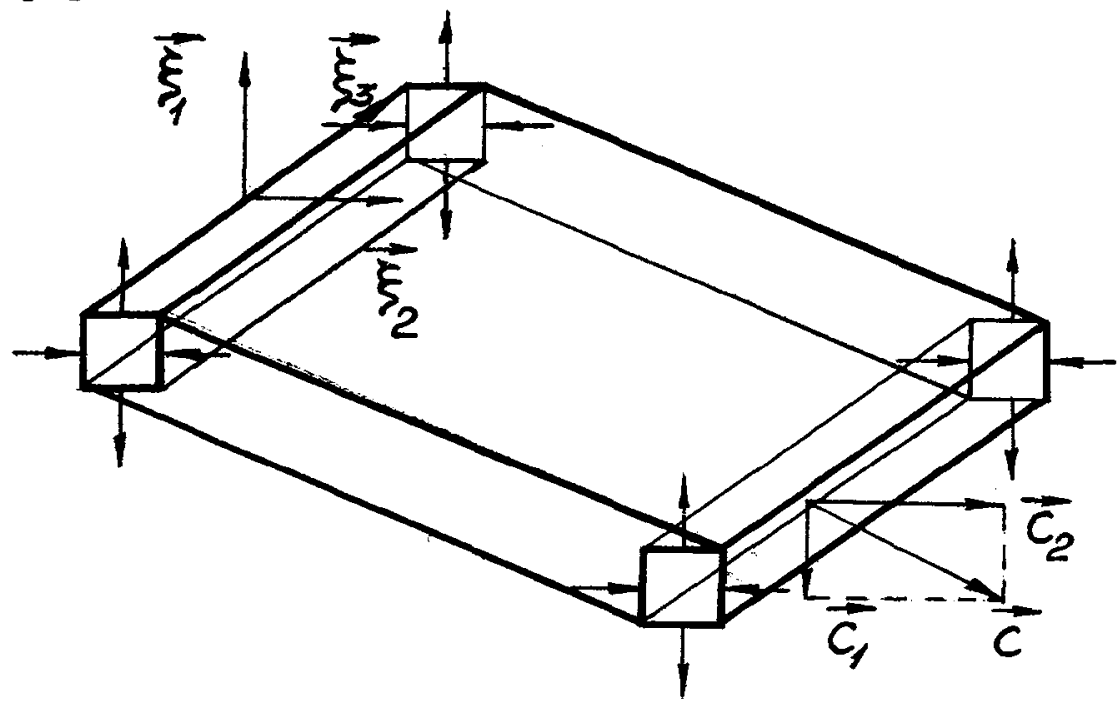

F1g.1. Scheme 1llustrating the martensitic lamella growth controlled by two longltudinal waves. 
the $\gamma-\alpha$ MT it is necessary to model the initial excited state. The last one should be parallelepipedal in form and well definitely orlented relative to the crystallographic axes of $\gamma$-phase lattice (the parallelepiped orientation is specified by the vectors $\vec{\xi}_{1}, \vec{\xi}_{2}, \vec{\xi}_{3}$ ). The cross dimensions d of such parallelepiped is defined from the characteristic thickmess of martensitic lamelia (about $1 \mu \mathrm{m}$ ), the oscillation frequency of $1 \mathrm{ts}$ opposite faces lies at the hypersonic frequency interval (10-100 $\mathrm{HHz})[6]$, the oscillation amplitude has to be sufficient to create the threshold deformation $\varepsilon^{\text {th }} \approx 0,001[7]$. It may be carried out e.g. by generation of two mutually orthogonal coherent waves with $1 \% 2 \lambda=\mathrm{d}$. The sound wave intensity I necessary for the MP initiation may be easy estimated: $I=2 \varepsilon^{t h} \mathrm{Gc} \approx 2 \mathrm{TW} / \mathrm{m}^{2}$, where $G$ (modulus of elasticity) $\approx 200 \mathrm{HN} / \mathrm{m}^{2}$ and $\mathrm{c}$ (velocity of longltudinal sound $\approx 5 \mathrm{~km} / \mathrm{s}$ are taken for the $\mathrm{Fe}-31,5 \% \mathrm{Ni}$ alloy [8]. In other words the wanted experiment on the sample with the work-surface area $10 \mathrm{~mm}^{2}$ needs a generator of hypersonic (10-100 HHz) osclilations with $2 \mathrm{MW}$ in power. Technical realization of mentioned parameters is possible by using the pulse sources only. It should be noted that the application of pulsed action is perfectiy Justifled because the one-time deformation of transformed volume is quite sufficient to initiate the MT in accordance with the condition (1). In this case the duration of acoustic pulse (5-50 ps) is defined by a half of period of controling osciliations. Further, the necessity of ultimate localization (to initiate the single $M C$ ) apparently keeps out the use of gas gun while it is the instrument available for the direct transformation of the kinetic energy to the sound osciliation energy. Besides, in this case it is difficult to expect the generation of hypersonic acoustic pulses with the amplitude sufficient to create the threshold deformation.

At present the problem of excitation of high-intensity plcosecond acoustic pulses by the supershort pulse laser can be considered as resolved one [9]. Application of the light radiation permits to concentrate an energy in the volume with dimensions about a wavelength (for a visible light $<1 \mu \mathrm{m}$ ) and to satisfy the theory demands.

The results of first successive experiments on the $\gamma-\alpha$ M Initiation in the $\mathrm{Fe}-31,5 \% \mathrm{N1}$ single crystals by unit laser pulse (pulse duration: $\approx 20 \mathrm{ps}$, wavelength: $0,63 \mu \mathrm{m}$, energy: $* 5 \mathrm{~mJ}$ ) are presented in [10]. The work face was parallel to the crystallographic plane $(001)_{\gamma}$ which had the smallest angle (about $2^{\circ}$ ) with the long axis $\vec{\xi}_{3}$ of martensite nucleus. In the same time the vector $\vec{\xi}_{1}$ is close to the direction [001 $]_{\gamma}$ and the angle $\varphi$ between $\vec{\xi}_{2}$ and the one of the four-fold axes lying in the plane $(001)_{\gamma}$ is about $17^{\circ}$. Since the wave normals $\mathbf{n}_{1,2}$ do not colncide in direction with the crystallographic symmetry axes the controlling waves are quasilongltudinal. The laser radiation was focused on the imradiated surface by a cyindrical lens therefor the laser action trace appeared as an elongated strip (the ratio of length to width about 500). The action area was similar in the form (taking into account a finite depth of radiation penetration) to the elongated parallelepiped. A geometry of experiment is shown on the Fig.2.

The conducted experiments have corroborated that the $\gamma-\alpha$ is may be initiated by the single picosecond laser pulse. On the other hand it was impossible to inltiate a transition for the slgnificant deviation of the specimen temperature $\mathrm{T}_{\mathbf{s p}}$ from the $\mathbf{M T}$ start temperature $\mathbf{M}_{\mathbf{s}}$. The typical difference value $T_{\mathbf{s p}}-\mathrm{M}_{\mathbf{B}} \approx 4 \mathrm{~K}$. Evidently, the warming of 
volume absorbing an energy (a favomable factor at the reverse $\alpha-\gamma$ MT) retards the $\gamma-\alpha$ MP initiation because the moving from $M_{s}$ is accompanied by the growth of the energetic interphase barrier.

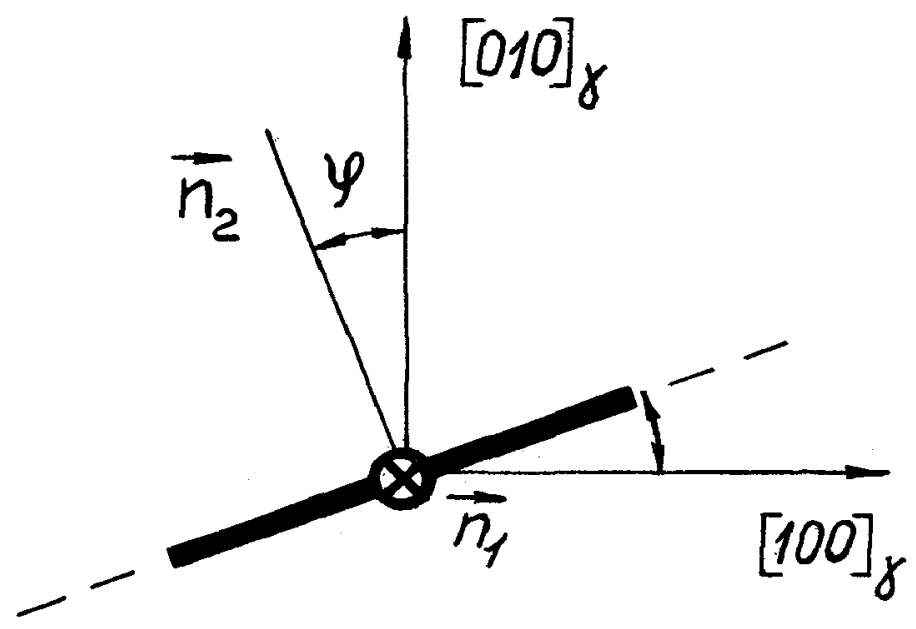

Fig.2. The orientation of the normals of the waves generated by the laser pulse. The bold line shows the laser trace. Sign $\otimes$ shows the orlentation of the wave normal $\mathbf{n}_{i} *[001]_{\gamma}$.

4. GENGRATION MECHANISUS OF CONIROLITNG WAVES AT THE IAASBR PUTSE ACTION The rectangular shape of the laser action trace on the tested specimen face allows to apply (for a qualitative interpretation) the analytical golution of problem on the indentation of the plane stamp which is infinite in length and finite in width [11]. The given problem is resolved within the scope of the elasticity dynamic theory for the predetermined law of the stamp movement. Fig.3 shows the fronts of waves excited by indented stamp in the half-unbounded isotropic medium occupying the half-space $z>0$ at some moment $t>0$. Deformation of volume under the stamp is defined by superposition of displacements which are created by the plane longltudinal wave $w_{p, 1}$, two cylindrical longituainal waves $\nabla_{0,1}$ and two cylindrical transverse waves $\nabla_{c, t}[11]$. The phase velocitles of longltudinal (plane and cylindrical) waves are equal and exceed ones of transverse waves. In the case of the pulse action $1 t$ is necessary to speak actually about acoustic pulses "p, $\mathbf{m}_{0,1}, \mathbf{W}_{0, t}$. A comparison of Fig.1. with Fig.3 shows that the initiation

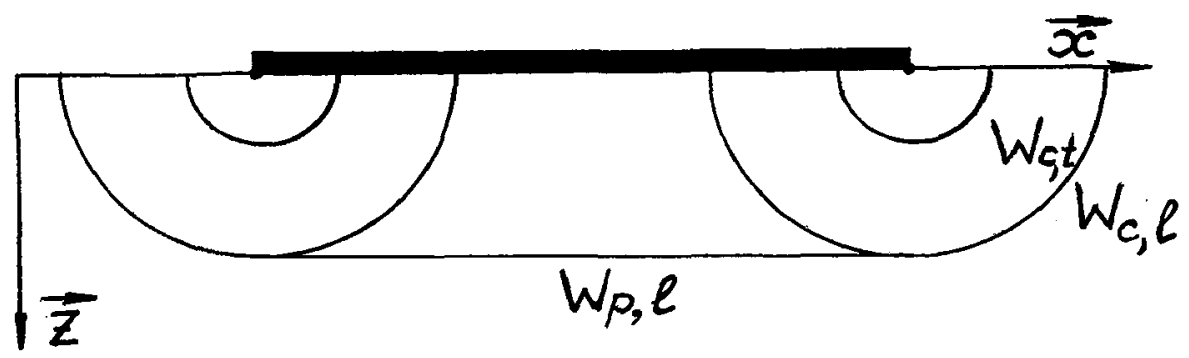

F1g.3. The Ironts of the waves excited by the moving plane stamp in the isotropic medium. The bold line shows the cross-section of the stamp. 
needs tension in the direction $\mathbf{z}$ and compression in the direction $\mathbf{z}$ (FIg.3). Areas with such deformation may be realized when the acoustic pulses $w_{p, 1}$ and $w_{0,1}$ are bipolar, $1 . e$. have both the compression stage and the tension one. Obviously, the tension deformation $\left(\varepsilon_{1}\right)$ has to be created by the pulse $\boldsymbol{W}_{\mathrm{p}, I}$ and the compression one $\left(\varepsilon_{2}\right)-$ by the pulse $\mathbf{T}_{0,1}$.

For cylindrical waves the compression and tension areas are known [12] to be obligatory. In the case of the plane wave the tension area is formed only after the compression pulse (formed by an external action) WIIl be reflected from the iree-stress specimen boundary.

The compression pulse is created in surface layer about $0,1 \mu m$ in thickness as result of both the thermoelasticity mechanism [9] and the recoll momentum of reflected photons and evaporated atoms (at the atom evaporation regime the contribution of the last mechanism is determinant). Certainly, the specimen surface may be regarded as iree one only after the Irradiation process will be completed.

Thus, In the case of the rectangular laser action trace there are two areas (near the specimen face) where the conditions demanded for the MC growth initiation may be realized.

The presented interpretation of creation of excited state by the laser action adjusts essentially the treatment proposed in works [5, 10] Where one of the two controlling waves was supposed to arise as a result of the Polsson's effect.

As an conclusion the following should be noted. According to the calculations $[13,14]$ it may be expected a stimulated initiation of martensite growth in the $\mathrm{Cu}-\mathrm{Zn}$ and $\mathrm{T} 1-\mathrm{N} 1-\mathrm{Cu}$ alloys by the pulse lager action. The positive results of similar experiments will give evidences for an extension of the theory conclusions [4] to the MT in alloys exhibiting the shape-memory effect.

\section{REFERENCES}

1.Sel Jakow N., Kurdjumor G. and Goodtzov N., Zeit. Phys. 45(1927) 384-408.

2. Lokshin F.I., Rate of Martens1t1c Transformation (Sclentiflc Reports of Higher School, Moscow: Metallurglya, 1958, No.2) pp.205-208.

3.Meshcheryakov Yu.I., Kashchenko M.P., Vasil kov V.B. and Atroshenko S.A., Pis'ma v Zhurnal Tekh. Fiz. 19(1993) 72-75.

4.Kashchenko M.P.', The wave model of martensite growth for $\gamma-a$ transformation in iron-based alloys (UIF Nauka, Ekaterinburg, 1993) 224p.

5. Kashchenko M.P. Ietuchev V.V., Konovalov'S.V. and Neskoromny 1 S.V., Phys. Met. Metailogr. $76(1993) 300-308$.

6.Vereshchagin V.P. and Kashchenko M.P., Fhys. yet. Metallogr.

61 (1986) $23-30$.

7. Kashchenko M.P., Phys. Met. Metallogr. 58(1984) 24-31.

8. Haush G. and Warrimont $\mathrm{H}$., Acta Wet. 21(1973) 401-414:

9. Gusev V.E. and Karabutov A.A., Iaser optoacoustics (Hoskow: Nauka, 1991) $304 \mathrm{p}$.

10.Letuchev V.V., Konovalov S.V., Neskoromny1 S.V. and Kashchenko M.P. J. Mat. Sc. Let. 11 (1992) 1683-1684.

11. Parton V. ${ }^{2}$. and Perlin P.I., Mathematical elasticity theory methods (Moskow: Nauka, 1981 ) p.p.473́-492.

12. Landau L.D. and Lifshits E.M., Continuous medium mechanics (Koskow: Nauka, 1954; $331-333$.

13 Arlstova N.V. Alexina I.V. and Kashchenko M.P., Fizika netaliov $t$ Hetal lovedente 77 (1994) 146-150.

14.Kashchenko M.P., Vereshchagin V.P. and Artstova N.V., Fizika Hetallov $i$ yetailovedenie 75 (1993) 38-43. 